\title{
Management and Prognostic Factors for Delayed Reconstruction of Neglected Posterior Shoulder Fracture-Dislocation
}

\author{
Taner Bekmezci ${ }^{1, *}$ and Egemen Altan ${ }^{2}$ \\ ${ }^{1}$ Department of Orthopaedics and Traumatology, Liv Hospital, Bahcesehir University, Istanbul, Turkey \\ ${ }^{2}$ Orthopaedics and Traumatology, Selcuk University, Konya, Turkey \\ ${ }^{*}$ Corresponding author: Taner Bekmezci, Department of Orthopaedics and Traumatology, Liv Hospital, Bahcesehir University, Istanbul, Turkey. Tel: +90-5052123260, Fax: +90-2122734400,
} E-mail:tanerbekmezci@hotmail.com

Received 2015 June 9; Accepted 2015 August 8.

\begin{abstract}
Introduction: Posterior fracture-dislocations of the shoulder are rare conditions. Misdiagnosis can occur in $50 \%-80 \%$ of the patients. Due to the size of the lesion, stability of the joint could not be achieved with transfer of subscapular tendon or tuberosity.

Case Presentation: A 54-year-old male patient was referred to our hospital with a neglected posterior shoulder fracture-dislocation. Functional results of the patient, and technical informations were explained in this case report. The reverse Hill-Sachs lesion involved $40 \%$ of the articular surface. Depressed and malunited fragment was elevated, and fixated to the humeral head.

Conclusions: Glenohumeral joint reduction with reconstruction of the humeral head seems to be possible even in a neglected locked posterior shoulder fracture-dislocation. If the depressed osteochondral fragment is still spheric, signs of the avascular necrosis are absent on CT, and malunion of the fragment has occurred, it is quite possible to reconstruct the head. Although results of the case are good, it is worthwhile to follow these patients for long-term complications.
\end{abstract}

Keywords: Reverse Hill-Sachs, Posterior Instability, Shoulder

\section{Introduction}

Posterior shoulder dislocations often occur after an electric shock or during seizure. Humeral head moves backward to the posterior of the glenoid due to the strong muscles which internally rotate and adduct (1). Misdiagnosis can occur in $50 \%-80 \%$ of the patients (2). Posterior edge of the glenoid can cause impaction of the anteromedial part of the humeral head; this is called a Reverse Hill-Sachs (RHS) lesion. Several surgical techniques including transfer of the subscapularis muscle, filling the defects with grafts, and arthroplasty may be performed (3-9). The described technique may be applied for neglected posterior fracture-dislocations of the shoulder which fulfills the suitable criteria for the RHS lesion.

To the best of our knowledge, reconstruction of a posterior shoulder fracture-dislocation that was neglected for a threemonth period has not been reported in the literature yet.

\section{Case Presentation}

A 54-year-old male patient had been admitted to the third stage medical center emergency room with a history of low-voltage electric shock trauma. He had suffered from arrythmia and severe pain and limited range of motion (ROM) of his right shoulder. This pa- tient has been determined and treated by a cardiologist. Orthopedic consultation had been completed after cardiac stability achieved by an orthopedic surgeon. Patient had been followed by conservative treatment including physiotherapy and medication.

Patient was admitted to our clinic after three months following trauma. He was complaining about pain, activity limitation of ROM. Visual analog pain score was 4 at rest and 9 at activity. Examination of the right shoulder revealed flexion of 60 degrees, abduction of 45 degrees, external rotation of 30 degrees, and internal rotation of posterior superior iliac spine. Constant shoulder score was 26 points. The neurologic status and circulation were determined as without any disorder.

Radiographic imaging indicated that glenohumeral conformity was lost. Computed Tomography (CT) measurements revealed a RHS lesion of approximately $40 \%$ of the humeral head and the lesser tuberosity was fractured and malunited to the head (Figure 1).

\subsection{Surgical Technique}

Under general anesthesia, the patient was positioned as beach chair. A deltopectoral incision was used and rotator interval was exposed. Biceps tenotomy was

Copyright (C) 2015, Kashan University of Medical Sciences. This is an open-access article distributed under the terms of the Creative Commons Attribution-NonCommercial 4.0 International License (http://creativecommons.org/licenses/by-nc/4.0/) which permits copy and redistribute the material just in noncommercial usages, provided the original work is properly cited. 
performed and tendon traction suture applied. The malunited lesser tuberosity was identified just medial side of biceps sulcus and osteotomy was performed. Capsule and the subscapularis muscle were not detached from the lesser tuberosity. Gentle reduction of the glenohumeral joint was performed; however, it was possible to observe that the humeral head was dislocating easily at 30 degrees of internal rotation (Figure 2).

Therefore, lesser tuberosity was shifted into the defect and fixated with temporary $\mathrm{K}$ wires. However, at this time, humeral head was dislocated at 50 degrees of internal rotation.

During exposure, we observed that the fractured and collapsed fragment was united to the metaphysial region, and unlikely, sphericity of the collapsed fragment was preserved. It was osteotomized and elevated to the original line of the articular surface. Fragment temporary fixation was performed with 2 K-wires. Humeral head rotational stability was achieved at this position until 90 degrees of internal rotation.

The lesser tuberosity was reduced just medial to the bicipital sulcus. In this position, external rotation was provided to be only 40 degrees. In order to avoid limitation of external rotation, lesser tuberosity was medialized on to the elevated articular fragment and fixed with two screws. The ROM was 140 degrees of forward flexion, 110 degrees of abduction, 70 degrees of external rotation at adduction, and 80 degrees of internal rotation at 90 degrees forward flexion. Biceps tenodesis was performed at the level of biceps sulcus with No. 2 Ethibond Excel (Ethicon, Somerville, NJ) suture as transosseous manner. Adequate hemostasis and surgical wound closure were than performed.

After surgery, the patient's shoulder was braced in a position of 30 degrees of abduction and neutral rotation with a sling. The patient was discharged from the hospital on the second post-operative day.

The patient's shoulder was immobilized for 6 weeks and codman exercises were initiated in the third postoperative week and active ROM was allowed at 6 weeks of surgery. Physical therapy continued for a total of 3 months.

At 5 years of follow-up, patient's ROM was 140 degrees of forward flexion, 110 degrees of abduction, 55 degrees of external rotation at adduction, and internal rotation at the L3 level. The patient has no pain with activity or at rest, and returned to his work without any limitation of motion after 3 months therapy. Activity of daily living was slightly restricted when trying to scratch him back with shoulder internal rotation. Constant score was revealed 80 points. Muscle strength tests (including Patte, Jobe and Lift-Off) were normal when comparing to contralateral shoulder.

Three-Dimensional CT of the patient showed that the lesser tuberosity was shifted $8.4 \mathrm{~mm}$ to the medial to the bicipital sulcus. (Figure 3 )
Figure 1. Locked Posterior Shoulder Dislocation (Pre and Postoperative Computed Tomography Measurements of Reverse Hill-Sachs)
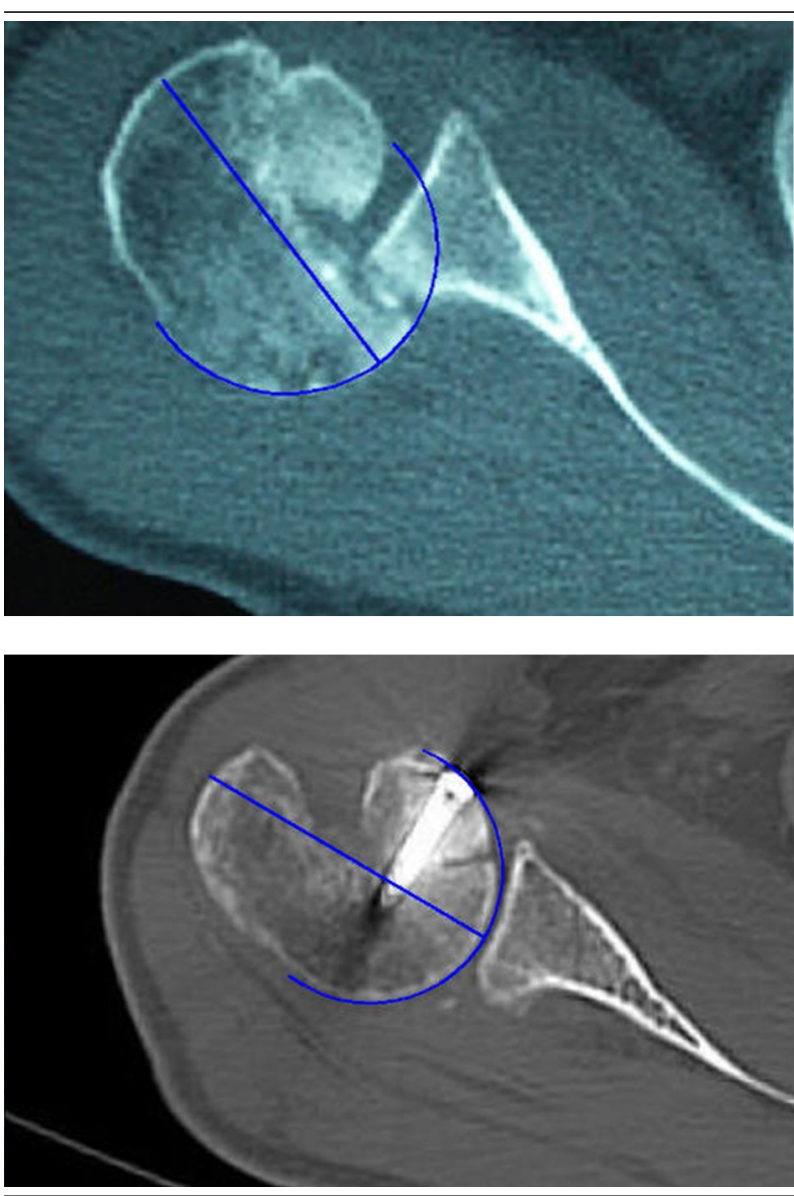

Approximately $40 \%$ of the articular surface was collapsed laterally and malunited to the metaphyseal region. This image was proportioned and scaled with the postoperative images at the same level of the humeral head to note how great is the articular fragment.

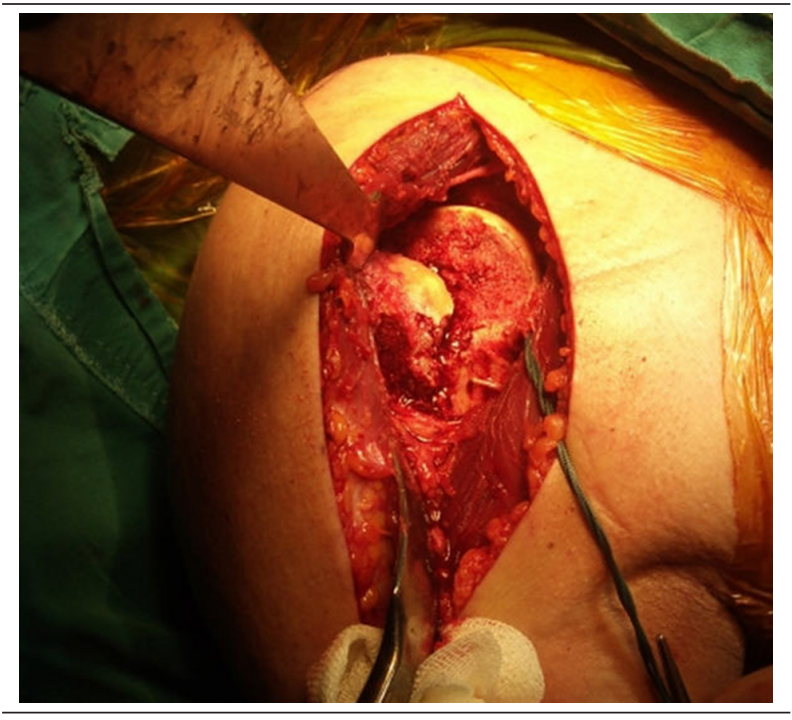

Figure 2. Following the Deltopectoral Approach, Glenohumeral Joint Was Reduced 

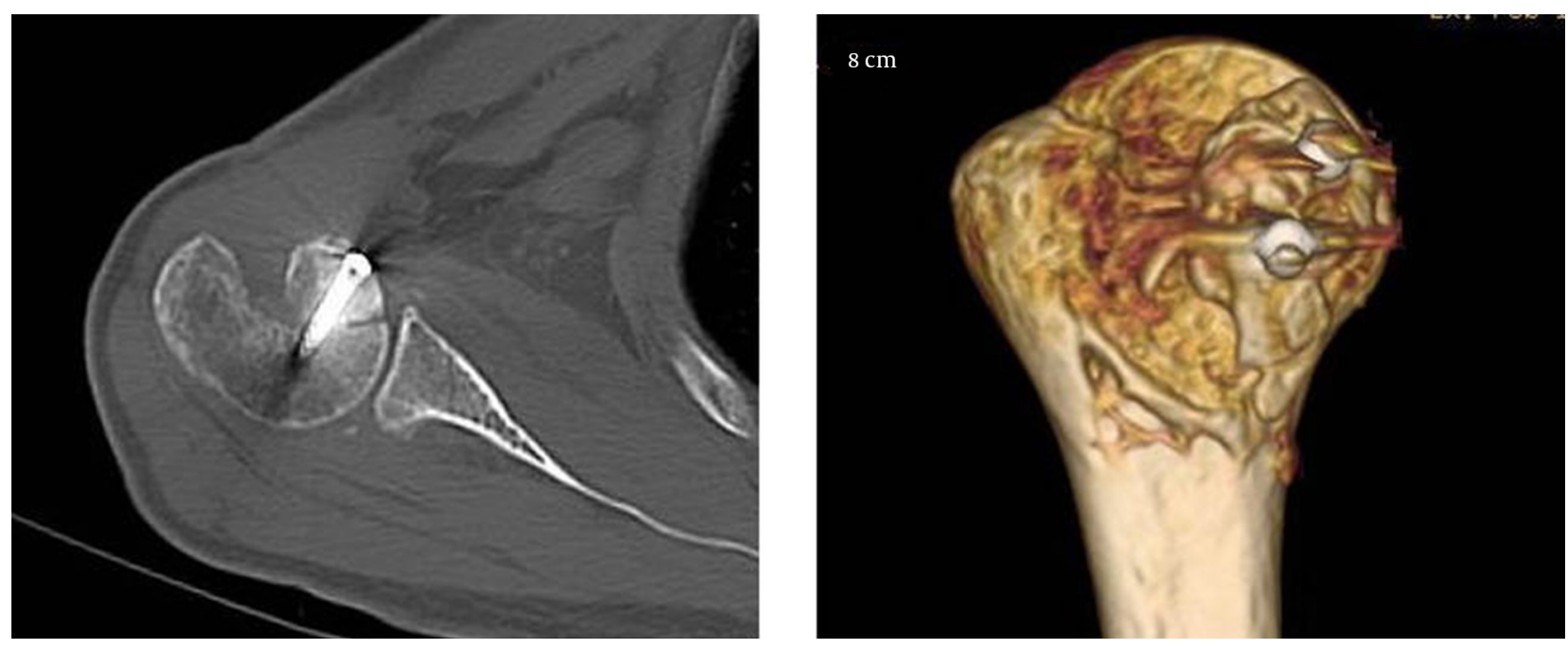

Figure 3. Computed Tomography and Three-Dimensional Reconstruction Views Demonstrate the Anatomical Fixation

\section{Discussion}

Management of neglected shoulder dislocation includes gaining normal ROM, and preventing the recurrences. Avascular necrosis and osteoarthrosis may also occur in the longterm $(4,6)$. Treatment of posterior shoulder dislocations varies depending on the extent of the lesion, age, and the time period between the diagnosis and the treatment. Closed reduction with sling immobilization had good results for acute dislocations accompanying with RHS lesions involving less than 20\% (4). McLaughlin reported subscapularis tendon transfer for lesions of more than $20 \%$ and less than $50 \%$ of the articular surface (7). Despite this technique, recurrence or osteoarthrosis has been reported (2).

In our case, the humeral head and associated fracture were evaluated with CT and measurements showed a locked posterior shoulder fracture dislocation with RHS lesion involving approximately $40 \%$ of the articular surface. Due to the size of the lesion, stability of the joint could not be achieved with transfer of subscapular tendon or tuberosity.

Gerber and Lambert advocated the use of allografts for significant articular defects to reconstruct the humeral head, and reported avascular necrosis in one of 4 patients (6). However, it is hard to get fresh allografts in our region and this makes difficult to perform this procedure on larger lesions. Disimpaction with grafting has been shown to be effective for defects of $<25 \%$ with good cartilage quality (10-12). Arthroplasty is the treatment of choice for those chronic dislocations with defects of larger than $50 \%$ of the articular surface accompanying with degeneration of the joint. Besides the technical difficulties and the complications due to the possible soft tissue imbalance, up to $60 \%$ successful rates have been reported $(2,4,13)$.

In contrast to other methods such as elevating the cartilage as a shell, we osteotomized the collapsed fragment and fixated with screws. Considering the limitation of motion caused by subscapularis muscle contraction, lesser tuberosity was fixated in a more medial position, preventing the loss of external rotation. The lesser tuberosity was transferred similar with the technique of McLaughlin and Neer modification, but neither the limitation of motion nor sub-scapularis muscle deficiency occurred $(2,7)$.

Although the time period between the initial trauma and the treatment is longer than 3 months, we obtained a satisfactory result which is an unusual condition that has not been reported in the literature yet. We are aware that our case is an extremely rare condition and we would like to emphasize the importance of this condition's characteristics which is an important factor in decision making process. Crosby et al. using tetracycline labeling for displaced three and four-part fractures, demonstrated a high rate of vascular preservation, especially in younger patients in the anterosuperior aspect of the humeral head (14). Previously defined area is consistent with the fractured fragment in our patient. It is possible to use the collapsed fragment to reconstruct the humeral head for the treatment of locked posterior shoulder dislocation, especially for those fragments that still prevent its sphericity. Our case has been earned us to be aware of that a fragment which was fractured and malunited to elsewhere may indicate a high suspicion for the vitality of the fragment leading to a high success rate for the reconstruction. Additionally, absence of signs of avascular necrosis during CT examination is a high evidence for viability of the fragment.

We suggest that such a neglected lesion must be evaluated carefully before surgery considering the previously mentioned factors. 


\section{Footnote}

Authors' Contribution:Study concept and design: Taner Bekmezci; acquisition of data: Taner Bekmezci; analysis and interpretation of data: Egemen Altan; drafting of the manuscript: Taner Bekmezci; critical revision of the manuscript for important intellectual content: Taner Bekmezci; statistical analysis: Egemen Altan; administrative, technical, and material support: Egemen Altan; study supervision: Taner Bekmezci.

\section{References}

1. Takase K, Watanabe A, Yamamoto K. Chronic posterior dislocation of the glenohumeral joint complicated by a fractured proximal humerus: a case report. J Orthop Surg (Hong Kong). 2006;14(2):204-7. [PubMed:16914790]

2. Hawkins RJ, Neer CS, Pianta RM, Mendoza FX. Locked posterior dislocation of the shoulder. J Bone Joint Surg Am. 1987;69(1):9-18. [PubMed: 3805075]

3. Delcogliano A, Caporaso A, Chiossi S, Menghi A, Cillo M, Delcogliano M. Surgical management of chronic, unreduced posterior dislocation of the shoulder. Knee Surg Sports Traumatol Arthrosc. 2005;13(2):151-5. doi: 10.1007/s00167-004-0524-6. [PubMed: 15756620]

4. Checchia SL, Santos PD, Miyazaki AN. Surgical treatment of acute and chronic posterior fracture-dislocation of the shoulder. $J$ Shoulder Elbow Surg. 1998;7(1):53-65. [PubMed: 9524341]

5. Altan E, Senaran H, Acar MA, Aydin K, Ozbaydar MU, Altan. Mozaicplasty Technique for Treatment of Reverse Hill-Sachs Lesion. Techniques in Shoulder \& Elbow Surgery. 2013;14(1):1-4.

6. Gerber C, Lambert SM. Allograft reconstruction of segmental defects of the humeral head for the treatment of chronic locked posterior dislocation of the shoulder. J Bone Joint Surg Am.
1996;78(3):376-82. [PubMed: 8613444]

7. McLaughlin HL. Locked Posterior Subluxation of the Shoulder: Diagnosis and Treatment. Surg Clin North Am. 1963;43:1621-2. [PubMed: 14090210]

8. Charalambous CP, Gullett TK, Ravenscroft MJ. A modification of the McLaughlin procedure for persistent posterior shoulder instability: technical note. Arch Orthop Trauma Surg. 2009;129(6):753-5. doi: 10.1007/s00402-008-0721-8. [PubMed: 18709379]

9. Diklic ID, Ganic ZD, Blagojevic ZD, Nho SJ, Romeo AA. Treatment of locked chronic posterior dislocation of the shoulder by reconstruction of the defect in the humeral head with an allograft. J Bone Joint Surg Br. 2010;92(1):71-6. doi: 10.1302/0301-620X.92B1.22142. [PubMed: 20044682]

10. Khayal T, Wild M, Windolf J. Reconstruction of the articular surface of the humeral head after locked posterior shoulder dislocation: a case report. Arch Orthop Trauma Surg. 2009;129(4):515-9. doi:10.1007/s00402-008-0762-z. [PubMed:18815798]

11. Bock P, Kluger R, Hintermann B. Anatomical reconstruction for Reverse Hill-Sachs lesions after posterior locked shoulder dislocation fracture: a case series of six patients. Arch Orthop Trauma Surg. 2007;127(7):543-8. doi: 10.1007/s00402-007-0359-y. [PubMed: 17522876]

12. Chalidis BE, Papadopoulos PP, Dimitriou CG. Reconstruction of a missed posterior locked shoulder fracture-dislocation with bone graft and lesser tuberosity transfer: a case report. JMed Case Rep. 2008;2:260. doi:10.1186/1752-1947-2-260. [PubMed: 18681972]

13. Gavriilidis I, Magosch P, Lichtenberg S, Habermeyer P, Kircher J Chronic locked posterior shoulder dislocation with severe head involvement. Int Orthop. 2010;34(1):79-84. doi: 10.1007/s00264009-0762-9. [PubMed:19300999]

14. Crosby LA, Finnan RP, Anderson CG, Gozdanovic J, Miller MW Tetracycline labeling as a measure of humeral head viability after 3- or 4-part proximal humerus fracture. J Shoulder Elbow Surg. 2009;18(6):851-8. doi: 10.1016/j.jse.2008.12.016. [PubMed: 19297204] 